\title{
NewSpace SAR: Disruptive Concepts for Cost-Effective Earth Observation Missions
}

Michelangelo Villano, Nertjana Ustalli, Luca Dell'Amore, Se-Yeon Jeon, Gerhard Krieger, and Alberto Moreira Microwaves and Radar Institute German Aerospace Center (DLR) Wessling, Germany michelangelo.villano@dlr.de

\author{
Maxwell Nogueira Peixoto \\ Aeronautics Institute of Technology \\ (ITA) \\ São José dos Campos, Brazil \\ maxwellpxt@gmail.com
}

\author{
Jan Krecke \\ Department of Electrical, Computer \\ and Software Engineering \\ University of Auckland \\ Auckland, New Zealand \\ jkre504@aucklanduni.ac.nz
}

\begin{abstract}
Synthetic aperture radar (SAR) is a key remote sensing technique for Earth observation. Future SAR missions will deliver weekly high-resolution images of our planet, thereby allowing quantification of several essential climate variables. While this is a huge step forward compared to current systems, some applications require even more frequent temporal sampling or simultaneous acquisitions from slightly different observation angles. NewSpace SAR denotes all groundbreaking solutions that enable frequent and enhanced SAR imaging at affordable costs. Besides the technological developments, e.g., mass-produced platforms for constellations of SAR satellites, application-driven SAR system design approaches play a fundamental role. Moreover, disruptive concepts based on formations of alternately-transmitting satellites, waveform or phase encoding and multi-focus-post processing, allow relaxing the design constraints, reducing complexity, size, and cost of the SAR instrument, and still retrieving the desired information from SAR data. These solutions will help spreading the on-going NewSpace revolution to SAR remote sensing and posing the basis for future Earth observation missions that will yield remarkable societal benefits.
\end{abstract}

Keywords-Synthetic aperture radar (SAR), NewSpace, waveform encoding.

\section{INTRODUCTION}

Synthetic aperture radar (SAR) is a remote sensing technique that exploits the Doppler shift arising from the sensor movement relative to the ground to improve the resolution in the flight direction well beyond the diffraction limit of the radar antenna. SAR therefore achieves highresolution imaging, while keeping an important feature of active microwave instruments, namely the ability to operate independently of weather conditions and sunlight illumination. Furthermore, the joint exploitation of multiple SAR images, acquired in different polarizations (polarimetric SAR), from slightly different observation angles (SAR interferometry, polarimetric SAR interferometry, and SAR tomography) and/or at different times (differential and permanent scatterer interferometry), allows retrieving a huge amount of unique information.

SAR is nowadays an established tool for Earth observation: Several satellites have been launched and operated as of 1978, and many airborne SAR systems have allowed early demonstrations of novel techniques, which have later been implemented in spaceborne missions [1]. More than 15 spaceborne SAR sensors are currently in operation, all launched within the last decade and characterized by a spatial resolution at least one order of magnitude higher than the sensors of the previous generation.
Among them, TerraSAR-X and TanDEM-X are the first satellites flown in a closely controlled formation to generate a seamless global digital elevation model with unprecedented accuracy and resolution [2]. State-of-the-art sensors also offer a much higher flexibility in that several acquisition modes can be selected for different trade-offs between resolution and coverage thanks to the use of phased array antennas with electronic beam steering. TerraSAR-X, for instance, can operationally achieve a resolution of $1 \mathrm{~m}$ in (sliding) spotlight mode or a $100 \mathrm{~km}$ swath width in ScanSAR mode, while its experimental modes allow an even higher azimuth resolution ( $0.2 \mathrm{~m}$ in staring spotlight mode), a wider swath (up to $260 \mathrm{~km}$ in wide ScanSAR mode), and dual- and fully-polarimetric acquisitions. TerraSAR-X data have been used to demonstrate several applications of SAR to environmental monitoring, such as the observation of the very fast movement of the Drygalski glacier, Antarctica, through thirty images acquired by TerraSAR-X during one year [Fig. 1 (a)], the mapping of a flood of the Mississippi River, USA [Fig. 1 (b)], the measurement of ground subsidence (up to $10 \mathrm{~cm}$ in four months) as a result of the water extraction in Mexico City, Mexico [Fig. 1 (c)], and the assessment of extensive logging in Mato Grosso, Brazil [Fig. 1 (d)]. In the latter case, clearings appear in the radar image as rectangular, relatively dark zones within the otherwise homogeneous surface of the forest; whereas covering large areas with optical cameras mounted on satellites is problematic in tropical regions due to the dense cloud layers.

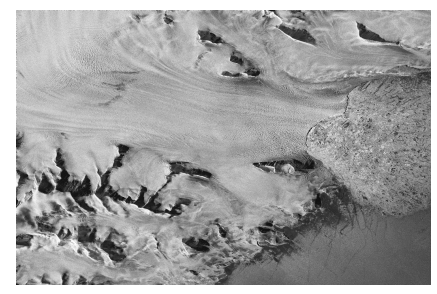

(a)

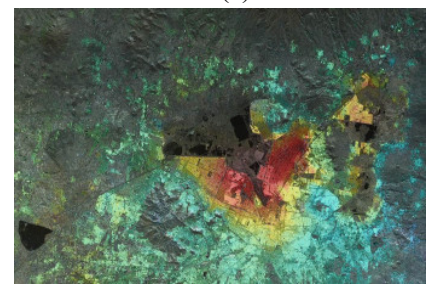

(c)

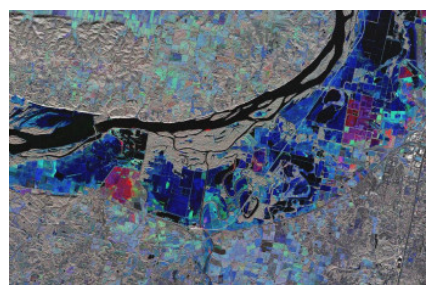

(b)

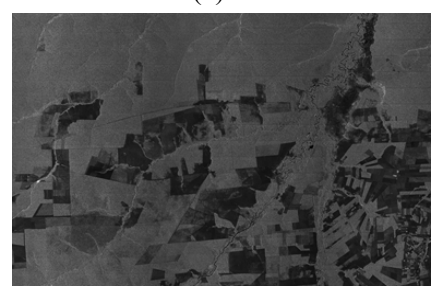

(d)
Fig. 1. Examples of applications of TerraSAR-X to environmental monitoring. (a) Observation of the Drygalski glacier, Antarctica. (b) Mapping of a flood of the Mississippi river, USA. (c) Measurement of ground subsidence in Mexico City, Mexico. (d) Assessment of extensive logging in Mato Grosso, Brazil. 
While very powerful and flexible, TerraSAR-X can map in stripmap mode only $2 \%$ of the Earth's landmass during its 11-day repeat cycle, due to its relatively small orbit duty cycle (the satellite can only operate 3 minutes per orbit) and its $30-\mathrm{km}$ swath width. Sensors launched more recently, such as Sentinel-1 and ALOS-2, are still characterized by comparable mapping capabilities at that resolution. This limitation is not due to technology development, but is inherent in the SAR acquisition principle. A brute force solution to this problem consists of flying a constellation of satellites on the same orbit, as done for Cosmo-SkyMed and Sentinel-1. This solution affords an increase in mapping capability by a factor equal to the number of satellites of the constellation, but becomes costly or even unfeasible, if the mapping capability has to be boosted by one or even two orders of magnitude.

\section{SAR MisSIONS FOR FREQUENT GLOBAL MAPPING}

In recent years there has been increased interest in the scientific community in understanding and quantifying dynamic processes within the Earth system occurring at different spatial and temporal scales, as well as their interdependency and interaction. Many of these processes are currently inadequately researched and understood. An important reason for this is the lack of suitable observation data for analyzing such interactions. Fig. 2 summarizes the requested observation intervals for the systematic monitoring of some exemplary dynamic processes on the Earth surface.

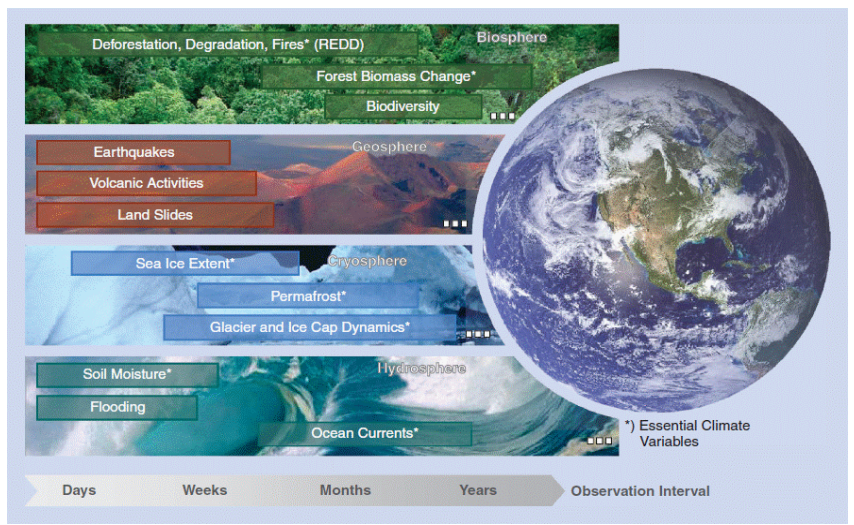

Fig. 2. Requested observation intervals for systematic monitoring of dynamic processes on the Earth surface.

The imaging performance and/or measurement resolution and accuracy of existing remote sensing configurations are often inadequate to draw reliable conclusions as to the dynamics of large-scale processes. The measurement of dynamic processes requires a continuous, extended and systematically planned observation strategy in order to detect changes and quantify them with sufficient accuracy. Depending on the processes to be observed, changes have to be measured on variable spatial and temporal scales and then related to one another. The combination of short revisit times and extended acquisitions over several years is required when it comes to accurate and high-resolution monitoring of fast developing, highly dynamic processes, such as the relaxation following an earthquake, as well as slowly developing processes, such as the inter-annual variation of forest biomass.

SAR represents the ideal candidate to provide answers to these questions, but spaceborne SAR sensors currently in operation do not have the resolution and mapping capability needed to meet these scientific requirements. In particular, a SAR sensor is required, capable of mapping the entire Earth surface twice a week in fully-polarimetric mode and with a spatial resolution below $10 \mathrm{~m}$ (this corresponds to a mapping capability two orders of magnitude better than that of TerraSAR-X in stripmap mode).

In response to these needs, a proposal for a highly innovative L-band SAR mission, Tandem-L, was started at DLR with a joint pre-phase A study with the National Aeronautics and Space Administration (NASA) in 2008 and has reached the phase B2 [3]. Tandem-L uses a deployable reflector antenna in combination with innovative digital beamforming (DBF) techniques. This increases the sensitivity and leads to a considerable reduction in transmit power. Because of this, the SAR instrument can be operated virtually continuously. DBF allows forming multiple elevation beams, which simultaneously map multiple subswaths. In this way, a resolution of $7 \mathrm{~m}$ over a $350 \mathrm{~km}$ wide ground swath can be achieved, as required for the aforementioned mission aimed at monitoring dynamic processes on Earth's surface [4]. A key element of the Tandem-L instrument is staggered SAR, a novel concept based on the continuous variation of the pulse repetition interval (PRI). The architecture with multiple elevation beams, in fact, yields "blind ranges" between the different subswaths. If the PRI is continuously varied, however, the ranges, from which the echoes are not received, because the radar is transmitting, will be different for each transmitted pulse. A proper selection of the PRIs together with an average oversampling of the signal in azimuth allows getting rid of the "blind ranges" and imaging a wide continuous swath with high resolution [5]-[7]. Staggered SAR is currently being considered as the base-line acquisition mode of the Tandem-L mission, where a strategy for onboard data volume reduction able to cope with the increased amount of data has also been devised [8].

Besides the Tandem-L proposal, the NASA-ISRO Synthetic Aperture Radar (NISAR) mission, the Radar Observatory System for Europe (ROSE-L), a future SAR mission of the European Space Agency's Copernicus Programme, and JAXA's ALOS-4 mission also aim at frequent global mapping with similar scientific objectives as Tandem-L [9].

\section{NewSPACE SAR: THE FUtURE OF SPACEBORNE SAR}

While the aforementioned missions represent a huge step forward by delivering vital missing information for improved scientific predictions upon which socio-political decisions can be based, several important applications would require an even more frequent, ideally daily temporal sampling, as is apparent from Fig. 2, or simultaneous imaging from slightly different observation angles, i.e., single-pass tomography.

The water exchange between land, ocean and atmosphere, for instance, is one of the most important factors determining weather and climate; hence, the accuracy of regional and global climate predictions is strongly dependent on the uncertainties in the dynamics of the water cycle. In climate models, soil moisture is a core parameter for characterizing the land surface, because it directly influences the heat and water transport between the land surface and the atmosphere. Even small variations in the water content have a large influence on the thermal characteristics of the land. 
Due to the strong temporal and spatial variability, measurement of soil moisture and its dynamics is an example of an essential variable, which would need to be recorded with temporal resolution much higher than that of TandemL, ideally daily. Other relevant climate variables, especially in the biosphere and geosphere, would also significantly benefit from an increased revisit time.

The existing concepts for high-resolution wide-swath imaging, however, cannot reach the requested coverage while keeping the resolution high, and the costs of increasing the mapping capability by launching and operating a constellation of satellites, as already pointed out, would be prohibitive. On the contrary, radically new concepts are needed that are revolutionary from the space segment pointof-view as well and provide easy and affordable access to space.

NewSpace SAR denotes all groundbreaking solutions that enable frequent and enhanced SAR imaging at affordable costs. Besides the technological developments, e.g., mass-produced platforms for constellations of SAR satellites, an application-driven SAR system design approach plays a fundamental role. Fig. 3 visualizes the main principles and goals of NewSpace SAR. While an interesting concept for a distributed and fractionated SAR system denoted as MirrorSAR was already proposed in [10], some disruptive concepts based on application-driven design, formation of alternately-transmitting SAR satellites, waveform or phase encoding and multi-focus-post processing are presented in the following that allow relaxing the SAR design constraints, reducing complexity, size, and cost of the SAR instrument, and still retrieving the desired information from SAR data.

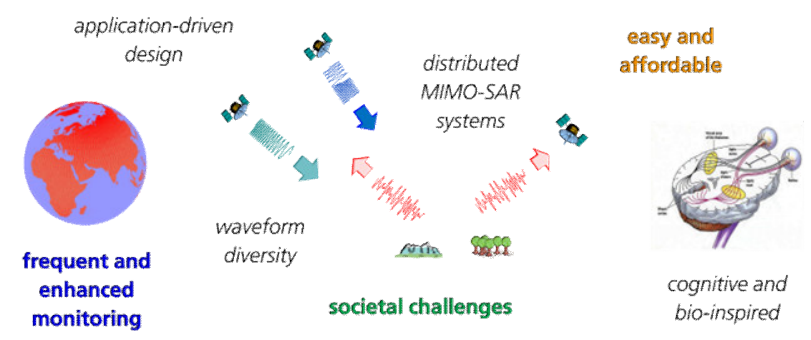

Fig. 3. Principles and goals of NewSpace SAR.

\section{A. Impact of ambiguity statistics on information retrieval}

In the design of SAR systems, the definition of the antenna height and the selection of the pulse repetition frequency (PRF) are driven by the need for controlling range and azimuth ambiguities. As ambiguities may impair the retrieval of information from SAR images, requirements are usually imposed on the ambiguity-to-signal ratio (ASR), i.e., on the total energy of the ambiguous signal relative to the total energy of the useful signal for an imaginary scene with uniform backscatter. Depending on the wavelength of the system, which determines different backscatter dynamics, a minimum ASR between $-20 \mathrm{~dB}$ and $-25 \mathrm{~dB}$ is typically required, where these values are purely based on the experience from existing SAR systems and are not linked to the consequential impairment of the retrieval performance within one or more specific applications.

Furthermore, the ASR requirement unfortunately does not account for the statistical distribution of the ambiguous signal that indeed plays a primary role in the way the retrieval of information is impaired. Observations from ambiguity simulations in staggered SAR and waveformencoded SAR, i.e., a SAR with pulse-to-pulse waveform variation, where the ambiguous signal is smeared and appears as a noise-like disturbance rather than as an ensemble of focused artifacts, suggest that ambiguous signals with the same energy but with different standard deviations might have very different effects on the retrieval of some parameters for some specific applications [6]-[7], [11]-[13]. In particular, a system with smeared ambiguities is likely to be more robust to applications based on polarimetric decompositions and related classifications and less affected by offsets due to ambiguities in cross-track interferometry [14].

As for the latter application, some preliminary results based on real TanDEM-X interferograms are shown in [15], where a coherent azimuth ambiguity has been simulated and added to the interferogram for conventional SAR (slightly defocused, attenuated, and shifted version of the main signal) and staggered SAR (fully smeared, attenuated, and shifted version of the main signal). Fig. 4 shows the interferometric phase (top) and the coherence magnitude (bottom), for (a) the ambiguity-free case (reference), (b) conventional SAR, and (c) staggered SAR, where for the latter two cases the
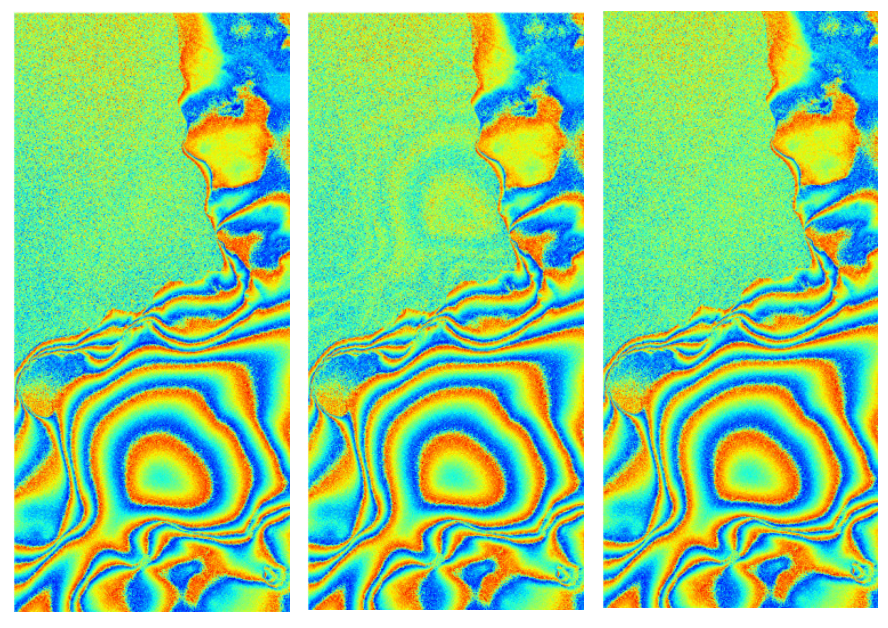

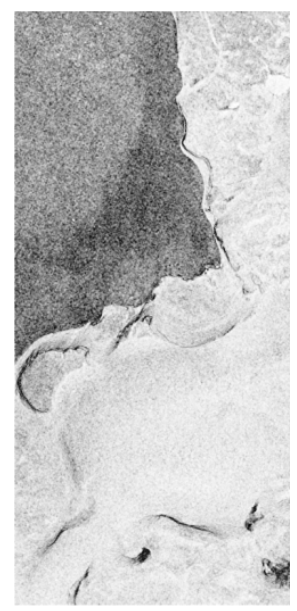

(a)

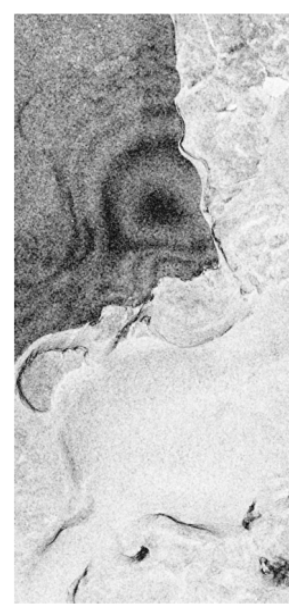

(b)

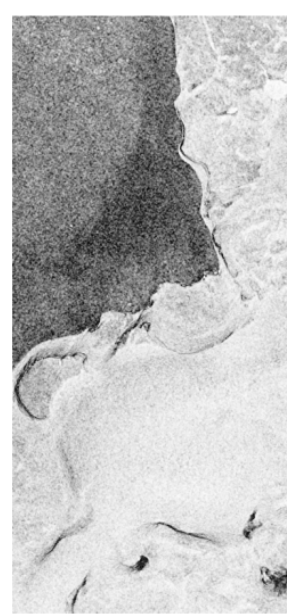

(c)
Fig. 4. Interferometric phase (top) and coherence magnitude (bottom) of TanDEM-X interferogram acquired over the Franz Josef Land, Russia. (a) Ambiguity-free interferogram. (b) Interferogram affected by azimuth ambiguities for conventional stripmap mode. (c) Interferogram affected by azimuth ambiguities for staggered SAR operation mode. The horizontal and vertical axes represent the slant range and azimuth, respectively. 
ambiguous signal has been attenuated in the simulation by $15 \mathrm{~dB}$ with respect to the main signal and is coherent between master and slave. In staggered SAR (Fig. 4 (c)), where the ambiguity is smeared, phase biases and coherence modulations are not observed, while they are present in conventional SAR (Fig. 4 (b)).

\section{B. Combination of waveform encoding and multi-focus post-processing}

Besides range and azimuth ambiguities a further constraint to account for within the design of SAR systems is represented by the nadir interference. While this is conventionally avoided by constraining the PRF selection, i.e., by choosing with the help of the timing (or diamond) diagram PRFs for which neither transmit nor nadir interferences occur, a novel concept based on the combination of waveform encoding and dual-focus postprocessing allow designing a SAR system without the nadir interference constraint and removing (not only smearing) the nadir echoes by means appropriate post-processing [11]. The acquired raw data are focused using a filter "matched" to the nadir echo, so that the nadir echo can be removed with a negligible corruption of the useful signal, as the nadir echo is focused and located at specific ranges, while the useful signal is smeared. Through an inverse focusing operation the latter focused data, in which the nadir echo has been removed, are then transformed back into raw data and finally focused using a filter that is "matched" to the useful signal, thus obtaining an image in which the nadir echo is significantly attenuated, while the useful signal is only minimally affected (Fig. 5).

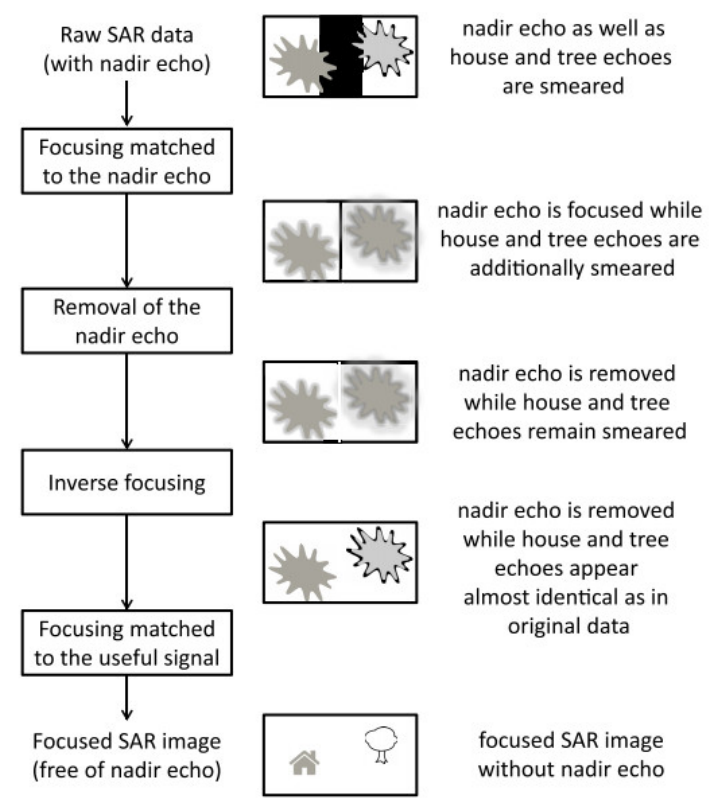

Fig. 5. Block diagram of dual-focus post-processing steps of waveformencoded SAR for nadir echo suppression (from [11]).

A validation of this technique through a dedicated TerraSAR-X experiment with up- and down- chirp alternation is currently being performed. In particular, a first TerraSAR-X data set was acquired over Tianjin, China, on 20th July, 2019. In particular, in the considered scenario a calm water surface is causing nadir interference on a town (Fig. 6) [16].

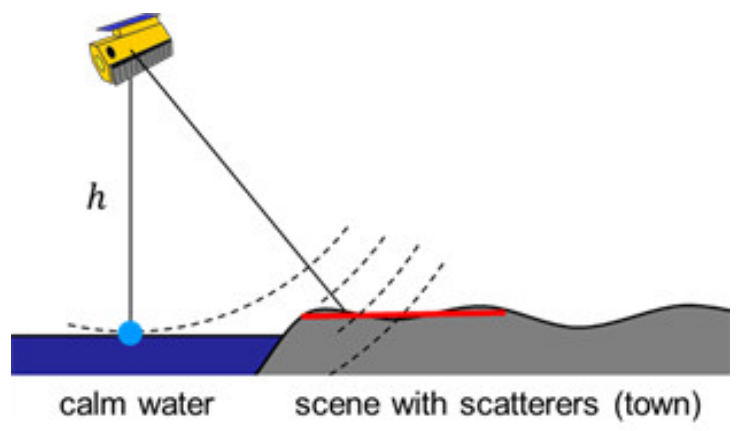

Fig. 6. Schematic representation of the scene selected for the TerraSAR-X experiment for validation of nadir echo suppression through up and- down chirp alternation and dual-focus post processing.

Multi-focus post-processing can also be exploited for range ambiguity suppression [12]-[13].

\section{Smallsats SAR for dedicated applications}

In the context of NewSpace SAR it is also of interest to understand how smallsats can be used for SAR. Following the discussion in Section 3.A, one possibility is to investigate what are the worst performance that can be tolerated for a dedicated application and design a SAR system able to retrieve the desired information without taking into account the visual appearance of the acquired images. In other words, the main challenge is to guarantee a satisfactory image quality in terms of signal-to-noise ratio (SNR) and ambiguities under the constraint of a reduced antenna size and available power. A typical example of application is ship detection, where the DLR Microwaves and Radar Institute has recently started a collaboration with the New Zealand Space Agency and the University of Auckland concerning the developing of a cost-effective SAR system to monitor illegal fishing and land deformation. Some first investigations are reported in [17].

\section{Formation of alternately-transmitting satellites}

While on the one hand smallsats could be employed for dedicated applications, where low SNR and ambiguities can be to some extent tolerated, on the other hand image quality comparable to that of current SAR satellites could be achieved through the exploitation of a formation of smallsats. In particular, an interesting concept is based on a formation of alternately-transmitting satellites, arranged in along-track, where each satellite transmits until the next one starts to transmit and all satellites receive the echoes of all transmitted pulses [18]. This allow reaching (ideally) a 100\% duty cycle and therefore lead to satisfactory noise equivalent sigma zero (NESZ) levels with moderate peak power. Furthermore, compared to concepts based on a single transmitter and several receive-only satellites, it provides intrinsic redundancy. This concept can be combined with waveform encoding and multi-focus post-processing for nadir echo and range ambiguity suppression.

\section{CONCLUSIONS}

This paper introduces NewSpace SAR as the ensemble of disruptive solutions that allow designing cost-effective Earth observation SAR missions. Novel techniques are discussed that exploit application-driven system design, waveform encoding, and smallsats, alone or flying in formation, depending on the imaging requirements. 


\section{REFERENCES}

[1] A. Moreira et al., "A tutorial on synthetic aperture radar," IEEE Geosci. Remote Sens. Mag., vol. 1, no. 1, pp. 6-43, Jan. 2013.

[2] G. Krieger et al., "TanDEM-X: A satellite formation for highresolution SAR interferometry," IEEE Trans. Geosci. Remote Sensing, vol. 45, no. 11, pp. 3317-3341, 2007.

[3] A. Moreira et al., "Tandem-L: A highly innovative bistatic SAR mission for global observation of dynamic processes on the Earth's surface," IEEE Geosci. Remote Sens. Mag., vol. 3, no. 2, pp. 8-23, Jun. 2015.

[4] S. Huber et al., "Tandem-L: a technical perspective on future spaceborne sar sensors for earth observation," IEEE Trans. Geosci. Rem. Sens., vol. 56, no. 8, pp. 4792-4807, Aug. 2018.

[5] M. Villano, G. Krieger, and A. Moreira, "Staggered SAR: high resolution wide-swath imaging by continuous pri variation," IEEE Trans. Geosci. Remote Sensing, vol. 52, no. 7, pp. 4462-4479, July 2014.

[6] M. Villano, , G. Krieger, M. Jäger, and A. Moreira, "Staggered SAR: performance analysis and experiments with real data," IEEE Trans. Geosci. Rem. Sens., vol. 55, no. 11, pp. 6617-6638, Nov. 2017.

[7] M. Villano, "Staggered synthetic aperture radar," $\mathrm{PhD}$ Thesis, Karlsruhe Institute of Technology, DLR-Forschungsbericht 2016-16, ISSN 1434-8454, Wessling, Germany, 2016.

[8] M. Villano, G. Krieger, and A. Moreira, "Onboard processing for data volume reduction in high-resolution wide-swath SAR," IEEE Geosci. Rem. Sens. Lett., vol. 13, no. 8, pp. 1173-1177, Aug. 2016

[9] P. A. Rosen et al., "Global persistent SAR sampling with the NASAISRO SAR (NISAR) mission," Proceedings of IEEE Radar Conference 2017, Seattle, WA, USA, 8-12 May 2017.

[10] G. Krieger et al., "Mirror-SAR: an advanced multistatic MIMO-SAR for high-resolution wide-swath Earth system monitoring," IEEE
International Geoscience and Remote Sensing Symposium (IGARSS) 2017, Fort Worth, Texas, USA, 23-28 July 2017.

[11] M. Villano, G. Krieger, and A. Moreira, "Nadir echo removal in synthetic aperture radar via waveform diversity and dual-focus postprocessing," IEEE Geosci. Rem. Sens. Lett., vol. 15, no. 5, pp. 719723, May 2018.

[12] M. Villano, G. Krieger, and A. Moreira, "Waveform-encoded SAR: a novel concept for nadir echo and range ambiguity suppression," Proc. European Conference on Synthetic Aperture Radar (EUSAR), Aachen, Germany, 5-7 June 2018.

[13] L. Dell'Amore, M. Villano, and G. Krieger, "Assessment of image quality of waveform-encoded synthetic aperture radar using real satellite data," Proc. International Radar Symposium 2019, Ulm, Germany, 26-28 June 2019.

[14] M. Villano, G. Krieger, "Impact of azimuth ambiguities on interferometric performance," IEEE Geosci. Rem. Sens. Lett., vol. 9, no. 5, pp.896-900, Sept. 2012.

[15] N. Ustalli and M. Villano, "Impact of ambiguity statistics on information retrieval for conventional and novel SAR modes", IEEE Radar Conference 2020, Florence, Italy, 21-25 Sept. 2020, unpublished.

[16] S.-Y. Jeon et al, "A TerraSAR-X experiment for validation of nadir echo suppression through waveform encoding and dual-focus postprocessing", European Conference on Synthetic Aperture Radar (EUSAR) 2020, in press.

[17] J. Krecke et al., "Design of smallsat SAR for dedicated New Zealand applications", European Conference on Synthetic Aperture Radar (EUSAR) 2020, in press.

[18] J. Mittermayer, P. Lopez-Dekker, T. Kraus, and G. Krieger, "Small satellite dispersed SAR - an exemplary configuration", Proc. European Conference on Synthetic Aperture Radar (EUSAR), Hamburg, Germany, 6-9 June 2016. 